\title{
A New cine-Substitution of Alkenyl Sulfones with Aryltitanium Reagents Catalyzed by Rhodium: Mechanistic Studies and Catalytic Asymmetric Synthesis of Allylarenes
}

Kazuhiro Yoshida and Tamio Hayashi*

Department of Chemistry, Graduate School of Science, Kyoto University, Sakyo, Kyoto 6068502, Japan

\section{Supporting Data}

General. All anaerobic and moisture-sensitive manipulations were carried out with standard Schlenk techniques under predried nitrogen or glovebox techniques under prepurified argon. NMR spectra were recorded on a JEOL JNM LA-500 spectrometer $\left(500 \mathrm{MHz}\right.$ for ${ }^{1} \mathrm{H}$, $76.5 \mathrm{MHz}$ for ${ }^{2} \mathrm{H}$, and $125 \mathrm{MHz}$ for ${ }^{13} \mathrm{C}$ ). Chemical shifts are reported in $\delta \mathrm{ppm}$ referenced to an internal $\mathrm{SiMe}_{4}$ standard for ${ }^{1} \mathrm{H} \mathrm{NMR}$, chloroform- $d(\delta 7.26)$ for ${ }^{2} \mathrm{H}$ NMR, and chloroform$d(\delta 77.05)$ for ${ }^{13} \mathrm{C}$ NMR. Optical rotations were measured on a JASCO DIP-370 polarimeter.

Materials. THF was distilled from sodium benzophenone-ketyl under nitrogen prior to use. Hexane was distilled from $\mathrm{Na} / \mathrm{K}$ alloy undn, s flask with a Teflon stopcock under nitrogen. Rhodium complex, $[\mathrm{Rh}(\mathrm{OH})((S) \text {-binap })]_{2}$ was prepared according to the reported procedure. ${ }^{1}$ Alkenyl sulfones, $(E)$-1-phenysulfonyl-1-octene $(\mathbf{1 a})^{2,3}, 2$-phenysulfonyl-1octene (1b), ${ }^{4}(E)$-1-phenylsulfonyl-2-phenylethene $(\mathbf{1 c}),{ }^{2,5}$ and 1-phenylsulfonyl-cyclohexene $(4)^{6}$ were prepared according to the reported procedures. 4-Bromoanisole, 4bromobenzotrifluoride, bis(cyclopentadieSupporting Information , 1-octyne, and benzenesulfonyl chloride were used as received. Chlorotriisopropoxytitanium ${ }^{7}$, bis(cyclopentadienyl)zirconium chloride hydride ${ }^{8}, 1$-deuterio-1-octyne ${ }^{9}$ were prepared according to the reported procedures.

Preparation of Aryltitanium Triisopropoxide (2). ${ }^{10}$ A typical procedure is given for the preparation of phenyltitanium triisopropoxide $\left(\mathrm{PhTi}(\mathrm{OPr}-i)_{3}\right)(\mathbf{2 m})$. To a solution of chlorotriisopropoxytitanium $(24.5 \mathrm{~g}, 94.0 \mathrm{mmol})$ in THF $(100 \mathrm{~mL})$ was added dropwise phenyllithium (100 mL, $0.94 \mathrm{M}$ in cyclohexane and diethyl ether, $94.0 \mathrm{mmol}$ ) over $5 \mathrm{~min}$ at $-78{ }^{\circ} \mathrm{C}$ under nitrogen. After completion of the addition, the cooling bath was removed and the mixture was stirred for $2 \mathrm{~h}$. The solution was concentrated in vacuo and dry hexane was added to the residue. The suspension was passed through celite to remove lithium chloride under nitrogen. Concentration of the filtrate and recrystalization from dry hot hexane to give the phenyltitanium triisopropoxide $(\mathbf{2 m})$ as a slightly yellow solid $(23.2 \mathrm{~g}, 76.8 \mathrm{mmol}, 82 \%$ yield $)$ which was stored in a glovebox.; $2 \mathrm{~m} ;{ }^{1} \mathrm{H}$ NMR $\left(\mathrm{CDCl}_{3}\right) \delta 1.25(\mathrm{~d}, J=6.3 \mathrm{~Hz}, 18 \mathrm{H}), 4.70$ (br septet, $J=6.3 \mathrm{~Hz}, 3 \mathrm{H}), 7.09-7.12(\mathrm{~m}, 3 \mathrm{H}), 7.51-7.56$ (br m, 2H). 4 Methoxyphenyltitanium Triisopropoxide (2n); ${ }^{1} \mathrm{H}$ NMR $\left(\mathrm{CDCl}_{3}\right) \delta 1.29(\mathrm{~d}, J=6.1$ $\mathrm{Hz}, 18 \mathrm{H}), 3.76(\mathrm{~s}, 3 \mathrm{H}), 4.63$ (br septet, $J=6.1 \mathrm{~Hz}, 3 \mathrm{H}), 6.66(\mathrm{~d}, J=8.0 \mathrm{~Hz}, 2 \mathrm{H}), 7.57(\mathrm{~d}, J$ $=8.0 \mathrm{~Hz}, 2 \mathrm{H})$; A solution of 4-methoxyphenyllithium was prepared by the slow addition of $n$-butyllithium in hexane to 4-bromoanisole in THF. 4-Trifluoromethylphenyltitanium Triisopropoxide $(20) ;{ }^{1} \mathrm{H}$ NMR $\left(\mathrm{CDCl}_{3}\right) \delta 1.25(\mathrm{~d}, J=6.1 \mathrm{~Hz}, 18 \mathrm{H}), 4.67$ (br septet, $J=$ $6.1 \mathrm{~Hz}, 3 \mathrm{H}), 7.35(\mathrm{~d}, J=7.8 \mathrm{~Hz}, 2 \mathrm{H}), 7.62(\mathrm{~d}, J=7.8 \mathrm{~Hz}, 2 \mathrm{H})$; A solution of 4trifluoromethylphenyllithium was prepared by the slow addition of $n$-butyllithium in hexane to 4-bromobenzotrifluoride in THF.

Preparation of $(E)$-2-Deuterio-1-phenysulfonyl-1-octene (1a-2-d $)$. To a solution of bis(cyclopentadienyl)zirconium dichloride $(2.92 \mathrm{~g}, 10.0 \mathrm{mmol})$ in THF $(19 \mathrm{~mL})$ was added a solution of $\mathrm{LiAlD}_{4}(84.0 \mathrm{mg}, 2.00 \mathrm{mmol})$ in THF $(3 \mathrm{~mL})$ at r.t. under nitrogen. The resulting mixture was allowed to stirred for $90 \mathrm{~min}$ and 1-octyne $(1.10 \mathrm{~g}, 10.0 \mathrm{mmol})$ was added. After $1 \mathrm{~h}$, benzensulfonyl chloride $(2.65 \mathrm{~g}, 15.0 \mathrm{mmol})$ was added and the mixture was heated to $40{ }^{\circ} \mathrm{C}$ for $3 \mathrm{~h}$. The mixture was diluted with hexane and was filtered through a short silica gel column $\left(\mathrm{Et}_{2} \mathrm{O}\right)$. Further purification by silica gel chromatography $($ Hexane/EtOAc $=$ 9/1) gave 1a-2- $d_{1}\left(1.53 \mathrm{~g}, 6.04 \mathrm{mmol}, 76 \%\right.$ yield).; ${ }^{2} \mathrm{H} \mathrm{NMR}\left(\mathrm{CHCl}_{3}\right) \delta 7.00$.

Preparation of $(E)$-1-Deuterio-1-phenysulfonyl-1-octene (1a-1-d $d_{1}$. To a solution of bis(cyclopentadienyl)zirconium chloride hydride $(1.55 \mathrm{~g}, 6.00 \mathrm{mmol})$ in THF (12 $\mathrm{mL})$ was added 1-deuterio-1-octyne $(447 \mathrm{mg}, 4.02 \mathrm{mmol})$ at r.t. under nitrogen. After $1 \mathrm{~h}$, 
benzensulfonyl chloride $(1.07 \mathrm{~g}, 6.03 \mathrm{mmol})$ was added and the mixture was heated to $40{ }^{\circ} \mathrm{C}$ for $3 \mathrm{~h}$. The mixture was diluted with hexane and was filtered through a short silica gel column $\left(\mathrm{Et}_{2} \mathrm{O}\right)$. Further purification by silica gel chromatography $($ Hexane/EtOAc $=9 / 1)$ gave 1a-1- $d_{1}$ (510 mg, $2.01 \mathrm{mmol}, 50 \%$ yield).; ${ }^{2} \mathrm{H}$ NMR $\left(\mathrm{CHCl}_{3}\right) \delta 6.34$.

Preparation of 2-Phenylsulfonyl-2-nonene (6). This was prepared from $\mathbf{1 a}$ according to the reported procedures; ${ }^{11}$ purified by PTLC (hexane) (74\% yield); Anal. Calcd for $\mathrm{C}_{15} \mathrm{H}_{22} \mathrm{O}_{2} \mathrm{~S}$ : C, 67.63; H, 8.32. Found: $\mathrm{C}, 67.66 ; \mathrm{H}, 8.19 . ;{ }^{1} \mathrm{H}$ NMR $\left(\mathrm{CDCl}_{3}\right) \delta 0.88(\mathrm{t}$, $J=7.0 \mathrm{~Hz}, 3 \mathrm{H}), 1.25-1.33(\mathrm{~m}, 6 \mathrm{H}), 1.48$ (quint, $J=7.4 \mathrm{~Hz}, 2 \mathrm{H}), 1.82(\mathrm{~s}, 3 \mathrm{H}), 2.18(\mathrm{q}, J=$ $7.5 \mathrm{~Hz}, 2 \mathrm{H}), 6.91(\mathrm{tq}, J=7.6,1.2 \mathrm{~Hz}, 1 \mathrm{H}), 7.52(\mathrm{t}, J=7.5 \mathrm{~Hz}, 2 \mathrm{H}), 7.60(\mathrm{t}, J=7.6 \mathrm{~Hz}$, $1 \mathrm{H}), 7.85(\mathrm{~d}, J=7.4 \mathrm{~Hz}, 2 \mathrm{H}) .{ }^{13} \mathrm{C} \mathrm{NMR}\left(\mathrm{CDCl}_{3}\right) \delta 11.46,13.99,22.49,28.19,28.37$, 28.85, 31.49, 127.96, 129.04, 133.04, 136.54, 139.38, 141.43.

General Procedure of Rhodium-Catalyzed cine-Substitution of Alkenyl Sulfones with Aryltitanium Reagents. $[\mathrm{Rh}(\mathrm{OH})((S) \text {-binap })]_{2}(4.5 \mathrm{mg}, 3.0 \mu \mathrm{mol})$ and alkenyl sulfone $(0.200 \mathrm{mmol})$ were placed under argon in a schlenk tube, and treated with a solution of $\operatorname{ArTi}(\mathrm{OPr}-i)_{3}(2)(0.400 \mathrm{mmol})$ in $\mathrm{THF}(0.5 \mathrm{~mL})$ at r.t. The schlenk tube was immersed in a bath maintained at $40{ }^{\circ} \mathrm{C}$ for $12 \mathrm{~h}$. The resulting mixture was cooled to r.t., treated with hexane (ca. $3 \mathrm{~mL}$ ) and $\mathrm{H}_{2} \mathrm{O}$ (ca. $50 \mu \mathrm{L}$ ), and filtered through a celite (eluent: hexane). After concentration of the filtrate, the residue was purified by PTLC on silica gel to give the corresponding product. 2-Phenyl-1-octene (3am): 12 purified by PTLC (hexane) (87\% yield); ${ }^{1} \mathrm{H}$ NMR $\left(\mathrm{CDCl}_{3}\right) \delta 0.87(\mathrm{t}, J=7.1 \mathrm{~Hz}, 3 \mathrm{H}), 1.21-1.35(\mathrm{~m}, 6 \mathrm{H}), 1.44$ (quint, $J$ $=7.5 \mathrm{~Hz}, 2 \mathrm{H}), 2.49(\mathrm{t}, J=7.7 \mathrm{~Hz}, 2 \mathrm{H}), 5.04(\mathrm{~d}, J=1.4 \mathrm{~Hz}, 1 \mathrm{H}), 5.25(\mathrm{~d}, J=1.4 \mathrm{~Hz}, 1 \mathrm{H})$, $7.25(\mathrm{t}, J=7.3 \mathrm{~Hz}, 1 \mathrm{H}), 7.31(\mathrm{t}, J=7.3 \mathrm{~Hz}, 2 \mathrm{H}), 7.40(\mathrm{~d}, J=7.8 \mathrm{~Hz}, 2 \mathrm{H})$. The geometry of the olefinic protons was assigned based on the following NOE data.

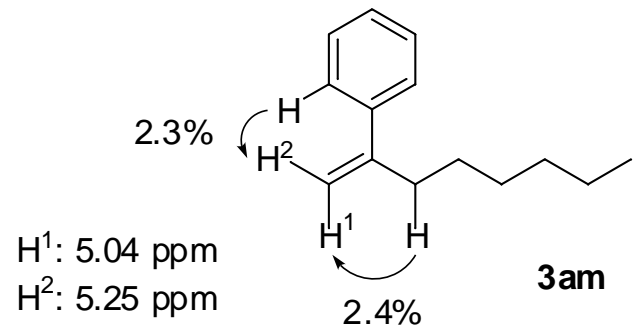

$(\boldsymbol{E})$-1-Deuterio-2-phenyl-1-octene $\left((\boldsymbol{E})-3 \mathrm{am}-\boldsymbol{d}_{1}\right)$ : purified by PTLC (hexane) (84\% yield); ${ }^{2} \mathrm{H}$ NMR $\left(\mathrm{CHCl}_{3}\right) \delta$ 5.04. ( $\left.\boldsymbol{Z}\right)$-1-Deuterio-2-phenyl-1-octene $\left((\boldsymbol{Z})-3 \mathrm{am}-\boldsymbol{d}_{1}\right)$ : purified by PTLC (hexane) (86\% yield); ${ }^{2} \mathrm{H}$ NMR $\left(\mathrm{CHCl}_{3}\right) \delta 5.25$. (E)-1-Phenyl-1-octene (3bm): ${ }^{13}$ purified by PTLC (hexane) (93\% yield). 1,1-Diphenylethene $(3 \mathrm{~cm}):{ }^{14}$ purified by PTLC (hexane) (99\% yield). 1-(4-Methoxyphenyl)-1-phenylethene (3cn): 15 purified by PTLC (hexane/AcOEt $=4 / 1)(99 \%$ yield). 1-(4-Trifluorophenyl)-1phenylethene (3co): 16 purified by PTLC (hexane) (97\% yield). (+)-( $\boldsymbol{R})$-3 Phenylcyclohexene (5m): ${ }^{17}$ purified by PTLC (hexane) $\left(94 \%\right.$ yield); $[\alpha]^{20} \mathrm{D}+147.7(c$ 1.00 , benzene) for $>99 \%$ ee. The enantiomeric purity was determined by GC analysis with chiral stationary phase column, CP-CHIRASIL-DEX-CB. The absolute configuration was determined to be $R$ by comparison of its specific rotation with the reported value. $18(+)-3-(4-$ Methoxyphenyl)cyclohexene (5n): 17 purified by PTLC (hexane/EtOAc $=10 / 1)(99 \%$ yield); $[\alpha]^{20} \mathrm{D}+145.8$ ( $c 1.00$, benzene) for $99.9 \%$ ee. The enantiomeric purity was determined by HPLC analysis with chiral stationary phase column, Chiralcel OB-H (hexane). (-)-3-(4-Methoxyphenyl)-1-nonene (7): purified by PTLC (hexane/Et $2 \mathrm{O}=20 / 1)(46 \%$ yield); Anal. Calcd for $\mathrm{C}_{16} \mathrm{H}_{24} \mathrm{O}: \mathrm{C}, 82.70 ; \mathrm{H}, 10.41$. Found: C, 82.53; H, 10.19.; ${ }^{1} \mathrm{H}$ NMR $\left(\mathrm{CDCl}_{3}\right) \delta 0.86(\mathrm{t}, J=7.2 \mathrm{~Hz}, 3 \mathrm{H}), 1.16-1.30(\mathrm{~m}, 8 \mathrm{H}), 1.60-1.71(\mathrm{~m}, 2 \mathrm{H}), 3.18(\mathrm{q}, J=7.5$ $\mathrm{Hz}, 1 \mathrm{H}), 3.78(\mathrm{~s}, 3 \mathrm{H}), 4.97-5.00(\mathrm{~m}, 2 \mathrm{H}), 5.88-5.95(\mathrm{~m}, 1 \mathrm{H}), 6.84(\mathrm{~d}, J=8.6 \mathrm{~Hz}, 2 \mathrm{H})$, $7.09(\mathrm{~d}, J=8.6 \mathrm{~Hz}, 2 \mathrm{H}) .{ }^{13} \mathrm{C} \mathrm{NMR}\left(\mathrm{CDCl}_{3}\right) \delta 14.12,22.68,27.52,29.30,31.82,35.52$, $49.03,55.25,113.48,113.79,128.47,136.79,142.93,157.88 . ;[\alpha]^{20} \mathrm{D}-27.0$ (c 1.00, $\mathrm{CHCl}_{3}$ ) for $99.2 \%$ ee. The enantiomeric purity was determined by HPLC analysis with chiral 
stationary phase column, Chiralcel OJ (hexane). For the determination of the enantiomeric purity, racemic samples of $\mathbf{5 m}, \mathbf{5 n}$, and $\mathbf{7}$ were also prepared by use of racemic binap.

\section{References} $124,5052$.

(1) Hayashi, T.; Takahashi, M.; Takaya, Y.; Ogasawara, M. J. Am. Chem. Soc. 2002,

(2) Duan, D.-H.; Huang, X. Synlett 1999, 317.

(3) Eisch, J. J.; Galle, J. E. J. Organomet. Chem. 1988, 341, 293.

(4) Hsiao, C.-N.; Shechter, H. J. Org. Chem. 1988, 53, 2688.

(5) Cardillo, G.; Savoia, D.; Umani-Ronchi, A Synthesis 1975, 453.

(6) Hopkins, P. B.; Fuchs, P. L. J. Org. Chem. 1978, 43, 1208.

(7) Dijkgraaf, C.; Rousseau, J. P. G. Spectrochim. Acta, Part A 1968, 24, 1213.

(8) Buchwald, S. L.; LaMaire, S. J.; Nielsen, R. B.; Watson, B. T.; King, S. M. Tetrahedron Lett. 1987, 28, 3895.

(9) den Reijer, C. J.; Drago, D.; Pregosin, P. S. Organometallics 2001, 20, 2982.

(10) (a) Rausch, M. D.; Gordon, H. B. J. Organomet. Chem. 1974, 74, 85. (b) Weidmann, B.; Widler, L.; Olivero, A. G.; Maycock, C. D.; Seebach, D. Helv. Chim. Acta 1981, 64, 357.

(11) Eisch, J. J.; Galle, J. E. J. Org. Chem. 1979, 44, 3279.

(12) Blatter, K.; Schlüter, A.-D. Synthesis 1989, 356.

(13) Negishi, E.-i.; Takahashi, T.; Akiyoshi, K. J. Organomet. Chem. 1987, 334, 181.

(14) Fleming, I.; Rowley, M. Tetrahedron 1986, 42, 3181.

(15) Guijarro, D.; Mancheño, B.; Yus, M. Tetrahedron 1993, 49, 1327.

(16) Bellucci, G.; Chiappe, C. J. Chem. Soc., Perkin Trans. 2 1997, 581. 2725 .

(17) Chung, K.-G.; Miyake, Y.; Uemura, S. J. Chem. Soc., Perkin Trans. 12000 ,

(18) Berti, G.; Macchia, B.; Macchia, F.; Monti, L. J. Org. Chem. 1968, 33, 4045. 